\title{
Challenges for management of post kala-azar dermal leishmaniasis and future directions
}

This article was published in the following Dove Press journal:

Research and Reports in Tropical Medicine

I4 November 2014

Number of times this article has been viewed

\author{
Dinesh Mondal' \\ Shinjiro Hamano ${ }^{2}$ \\ Md Golam Hasnain' \\ Abhay R Satoskar ${ }^{3}$ \\ 'Centre for Nutrition and Food \\ Security, Parasitology Laboratory, \\ (icddr, b) Mohakhali, Dhaka, \\ Bangladesh; ${ }^{2}$ Department of \\ Parasitology, Institute of Tropical \\ Medicine (NEKKEN), Nagasaki \\ University, Nagasaki, Japan; \\ ${ }^{3}$ Departments of Pathology and \\ Microbiology, Wexner Medical \\ Center, The Ohio State University, \\ Columbus, $\mathrm{OH}$, USA
}

Correspondence: Dinesh Mondal Centre for Nutrition and Food Security, Parasitology Laboratory, ICDDR, B, Mohakhali, Dhaka 1212, Bangladesh Fax +88029827101

Email din63d@icddrb.org

\begin{abstract}
Post kala-azar dermal leishmaniasis (PKDL) is a skin complication resulting from infection with Leishmania donovani (LD) parasite. It mostly affects individuals who have previously suffered from visceral leishmaniasis (VL) caused by LD. In some cases, PKDL develops among people infected with LD, but do not show any symptoms of VL. Clinical presentation includes hypopigmented macules/papules/nodules or polymorphic lesions (combination of two or more lesions). Except for skin lesions, PKDL patients are generally healthy and usually do not seek medical care. These patients play an important role in interepidemic transmission of the infection and subsequent VL outbreak. Therefore, proper diagnosis and treatment of PKDL patients is important for the control of VL in endemic countries, especially in the Indian subcontinent where VL is anthroponotic. Here, we report the challenges in the estimation of PKDL burden, its diagnosis, and treatment, and suggest possible solutions based on recent literature, reports, published manuals, and web-based information.
\end{abstract}

Keywords: post kala-azar dermal leishmaniasis, Bangladesh, challenge, treatment, diagnosis, prevention

\section{Challenges for post kala-azar dermal leishmaniasis case management Differences in incidence and clinical presentation depending on geographical areas}

Post kala-azar dermal leishmaniasis (PKDL) is a skin manifestation of infection caused by Leishmania donovani (LD) parasite. ${ }^{1}$ It develops mostly in patients who have been previously cured from visceral leishmaniasis (VL). ${ }^{1}$ Thus, it is considered as a complication of VL. However, several studies demonstrated that PKDL developed among individuals who were infected with LD without developing VL. ${ }^{2,3}$ Fortunately, these are observed in only a small proportion of PKDL cases $(6 \%-10 \%))^{2,3}$ Para-PKDL are cases in which patients have VL and PKDL at the same time. ${ }^{4}$ Para-PKDL is rare in the Indian subcontinent and is seen among $16 \%$ of PKDL cases in Sudan. ${ }^{5}$ PKDL can also be caused by Leishmania infantum in HIV-infected patients. ${ }^{4}$ In the Indian subcontinent, PKDL was first described by Brahmchari in 1922 as "dermal leishmanoid" because LD bodies had been observed in lesion smears. Later, the condition was renamed as post kala-azar dermal leishmaniasis. ${ }^{6}$ In Sudan, Christopherson first described PKDL in $1921 .^{7}$

Skin manifestations of PKDL include hypopigmented macular/papular/nodular or polymorphic lesions as well as a combination of these conditions. Involvement 
of the mucosa and other associated symptoms such as itching are rare. ${ }^{1}$ Interestingly, the clinical manifestation of PKDL shows regional characteristics. Sudanese with PKDL presents mostly nodular lesions, whereas most of the PKDL cases in Bangladesh show hypopigmented macular lesions $^{1-3,8}$ (Figures 1-3). Reasons behind this interesting phenomenon in the presentation of the disease are obscure. Apart from skin lesions, PKDL patients are clinically healthy and can perform daily activities., ${ }^{2,3}$ This explains why these patients usually do not seek medical care, and they remain unnoticed by the health system if they have not been actively researched. PKDL patients, especially those with papules and nodules, continue to transmit the infection to others through sandfly bites. Studies in the past have successfully demonstrated that the parasites can be transmitted from papular and nodular lesions through sandfly bites. ${ }^{9}$ However, it remains to be determined whether PKDL can also be transmitted from hypopigmented macular lesions. Theoretically, they should be because in $45 \%$ of clinically defined PKDL cases with hypopigmentation, their lesions were shown to have parasite DNA. ${ }^{1,8}$ Parasite DNA was also isolated from the peripheral blood of PKDL cases with macular lesions. ${ }^{8}$ Nevertheless, experimental xenodiagnosis is ultimately required to understand the potential of LD transmission through macular lesions of PKDL. Underlying host and parasite factors that make PKDL capable of transmitting infection are very important from a public health perspective.

Current literature shows that PKDL is most common in Sudan and Bangladesh. ${ }^{10}$ PKDL also occurs among cured VL patients in India, Nepal, and Ethiopia. However, the incidence is less in these countries than in Sudan and Bangladesh. ${ }^{10}$ PKDL incidence among sodium

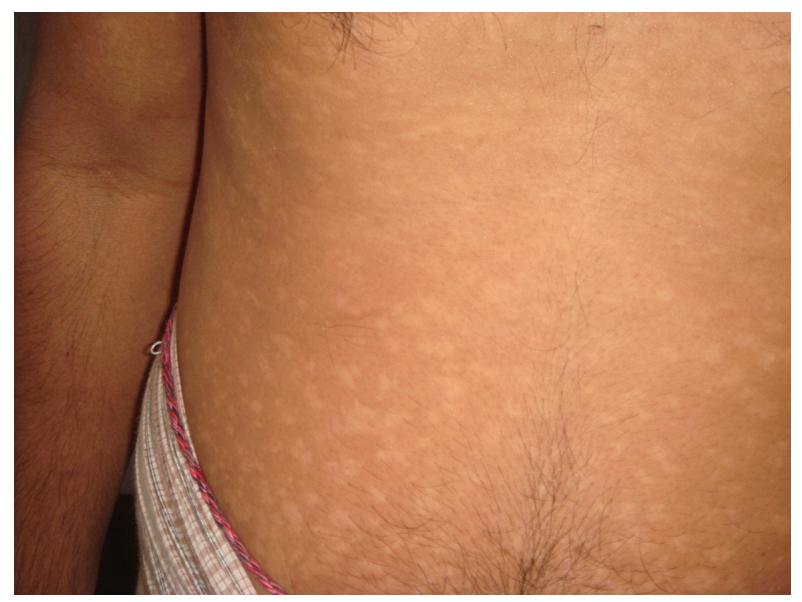

Figure I Macular form of post kala-azar dermal leishmaniasis.

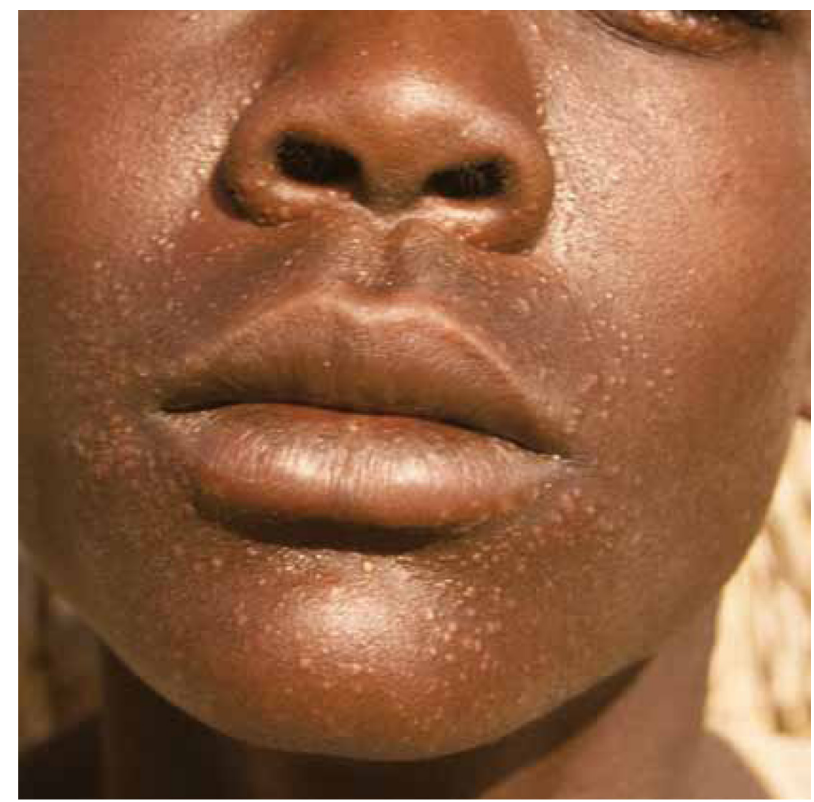

Figure 2 Papular post kala-azar dermal leishmaniasis.

Notes: Reproduced from The Post Kala-azar Dermal Leishmaniasis Atlas: A manual for health workers. Zijlstra EE, Alvar J. Geneva: World Health Organization. WHO/HTM/ NTD/IDM/20 I2.4. (c) 20I2. Available from: http://apps.who.int/iris/bitstream/l0665/ 101164/1/9789241504102_eng.pdf. Accessed October 3, 2014."

stibogluconate (SSG)-treated VL patients is about $50 \%$ in Sudan within 6 months after treatment. In Bangladesh, the incidence of PKDL from VL after treatment with SSG is about $19 \%$ within 5 years after treatment. ${ }^{3}$ The median time for the incidence of PKDL in India is about 2 years from the time of treatment. ${ }^{2,8}$ It has been speculated that the development of PKDL could be linked to treatment with SSG/ antimonials. ${ }^{12}$ Since antimonials have been the only drugs in use for the treatment of VL for almost 70 years, it is difficult

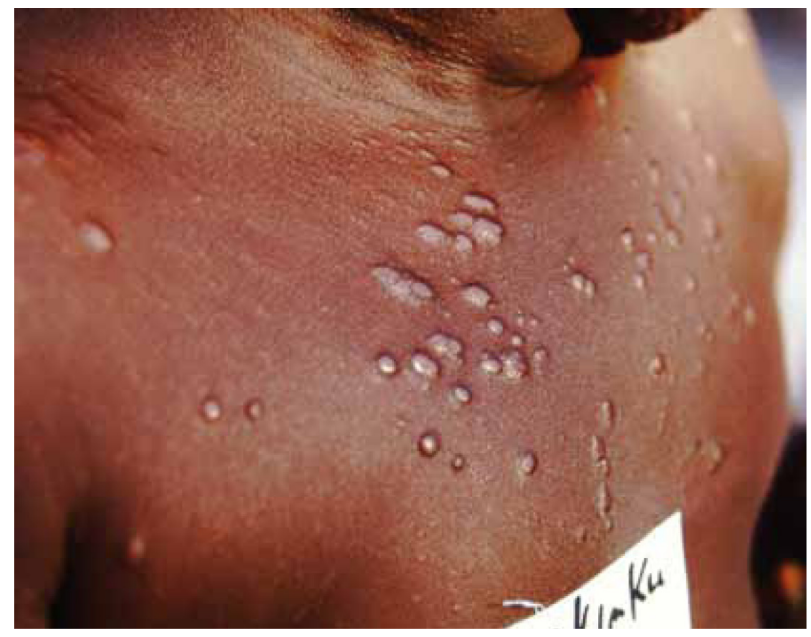

Figure 3 Nodular papular post kala-azar dermal leishmaniasis.

Notes: Reproduced from The Post Kala-azar Dermal Leishmaniasis Atlas: A manual for health workers. Zijlstra EE, Alvar J. Geneva: World Health Organization. WHO/HTM/ NTD/IDM/2012.4. (c) 2012. Available from: http://apps.who.int/iris/bitstream/l0665/ I01164/1/9789241504102_eng.pdf. Accessed October 3, 2014." 
to simply link antimonials with PKDL. Furthermore, recent reports demonstrated that PKDL developed after treatment of VL with other drugs..$^{13,14}$ Current available options for treatment of VL are miltefosine, paromomycin, liposomal amphotericin B, and amphotericin B deoxycholate. These could be used alone or in combinations: amphotericin $\mathrm{B}$ deoxycholate with miltefosine/paromomycin, miltefosine with liposomal amphotericin B, or paromomycin with liposomal amphotericin. ${ }^{15}$ If some of these treatment regimens are found to be protective against PKDL, it will be good news for public health policymakers. Therefore, either retrospective or prospective studies to explore the incidence of PKDL in relation to the various treatment regimens are needed especially in the Indian subcontinent where VL is only anthroponotic.

During the period of 1960-1970, VL disappeared from Bangladesh as a result of DDT (dichlorodiphenyltrichloroethane) sprays for malaria eradication. ${ }^{16}$ It subsequently re emerged in the early 1980 s as reported in the literature, first for PKDL cases, followed by reports of VL outbreaks. ${ }^{17-20}$ A recent report from Assam, India, showed that the outbreak of VL occurred in the same areas where VL had been a serious public health problem 70 years ago. ${ }^{21}$ Theoretically, the existence of a single case of PKDL can be a risk for new outbreak of VL. Thus, a complete survey of the absolute burden of PKDL in VL-affected countries is essential. Although there are some reports about the incidence of PKDL in the Indian subcontinent and in Africa, there is no survey for the estimation of PKDL burden with case mapping in VL endemic countries. The report from Assam further emphasizes the need for such a study as well as a thorough surveillance of VL-affected areas. ${ }^{21}$ It is evident that mapping of past and current VL as well as untreated PKDL cases is important for effective monitoring and to strengthen $\mathrm{VL}$ control strategies.

\section{Diagnosis}

The diagnosis of PKDL remains a big challenge for experts, especially in the macular form of the disease. ${ }^{10,22} \mathrm{LD}$ parasite can be demonstrated in $90 \%$ of skin specimens of cases with nodular lesions. Unfortunately, in macular cases, the sensitivity of conventional microscopic examination of skin specimens is only about $3 \% .{ }^{8,22,23}$ Adams et al ${ }^{24}$ reviewed and assessed the sensitivity and specificity of existing diagnostic tools for PKDL but failed to estimate the sensitivity of macular type PKDL. As such, the diagnosis of PKDL still relies on clinical criteria that include history of VL/exposure to VL endemic areas, skin lesions, and positivity for rK39 strip test combined with the exclusion of diseases like leprosy and fungal skin infection. 2,3,8,10,24 There is therefore an urgent need for new diagnostic tool(s) with high sensitivity and specificity for PKDL.

\section{Treatment}

The most important challenge for management of PKDL is the treatment of PKDL. Until now, SSG is the only option for the treatment of PKDL. ${ }^{25}$ Treatment includes intramuscular injection with SSG at a dose of $20 \mathrm{mg} / \mathrm{kg} /$ day for 20 consecutive days per month for 6 months (120 injections in total). Injections with SSG are very painful and serious adverse events including death from sudden cardiac arrest have been observed. ${ }^{26,27}$ Thakur et $\mathrm{al}^{27}$ in their observational study with $80 \mathrm{VL}$ patients with SSG treatment observed different types (abnormal P, R, T waves; elevation/depression of ST segment; and prolonged QT interval) of cardiac toxicity in 6\%-40\% of cases and death due to cardiac toxicity among 5\%. Other minor side effects included loss of appetite, metallic taste in mouth, and arthralgia. Other studies also reported SSGrelated cardiac toxicties. ${ }^{28,29}$ This is one of the reasons why PKDL patients usually do not seek medical care, and treatment compliance is frequently low when they receive SSG treatment. ${ }^{8}$ Thus, a safe, effective, and affordable treatment regimen for PKDL with shorter duration is urgently needed. Otherwise, PKDL patients will be left untreated and this will jeopardize the efforts of the national VL elimination program in Bangladesh and in the Indian subcontinent.

Alternative drugs for PKDL are miltefosine, amphotericin $\mathrm{B}$, liposomal amphotericin $\mathrm{B}$, and immunotherapy in combination with SSG. Thakur et $\mathrm{al}^{30}$ compared the efficacy of amphotericin B with SSG and found that amphotericin B was superior to SSG for the treatment of PKDL patients in India. However, the nephrotoxicity caused by amphotericin B coupled with high costs have limited its use. ${ }^{30}$ The results of the trial using liposomal amphotericin B for the treatment of PKDL by Médecins Sans Frontières, Holland program in Fulbaria, Bangladesh, showed that $90 \%$ had some response to treatment and 34\% had complete resolution of skin lesions by 12 months after treatment. ${ }^{10}$ Another alternative for SSG therapy is the combination of SSG with immunotherapy. So far only one study reported a high cure rate $(87 \%)$ of PKDL with immunotherapy in Sudan. ${ }^{31}$ The immunotherapy was based on the combination of SSG with the first-generation vaccine (alum-precipitated autoclaved Leishmania major plus Bacillus Calmette -Guérin). However, the risk for serious adverse events from SSG and autoclaved $L$. major cannot be eliminated. 


\section{Future directions to improve PKDL case management Improve PKDL case detection}

As mentioned earlier, PKDL patients especially in the Indian subcontinent are otherwise healthy except for skin lesions and they usually do not self-report to the hospital for medical care. Therefore, active detection of PKDL cases either by periodic camp approach or house to house visit of past VL cases is useful for the detection and treatment of PKDL cases. Huda et $\mathrm{al}^{32}$ in their multicenter study reported that PKDL case detection can be improved by $40 \%$ by implementing the camp approach. To reduce transmission of VL, the national VL elimination program in the Indian subcontinent is conducting indoor residual spraying with insecticides in houses of VL endemic villages at least twice. Before spraying, the spray team is required to perform a household visit to instruct the household head and members to take some actions and precautions. The spray team leader is usually a healthcare worker from the hospital. This provides an excellent opportunity to implement active case detection for PKDL if the healthcare worker is trained in the detection method. Another option is heath education to VL patients during treatment for $\mathrm{VL}$ in the hospital. If a VL patient is educated about PKDL, especially its clinical symptoms and treatment options by his/her doctor or nurse, then it may improve self-reporting of PKDL patients to the hospital for treatment. Another opportunity is to ensure periodic follow-up of treated VL patients for PKDL by providing incentives against his/her daily wage loss and by providing transportation to the hospital using the health system or a public-private partnership. Implementation research regarding any of above-mentioned options may help to establish the most cost-effective method for improving PKDL case detection. Support from donor agencies will be needed since PKDL is highly neglected among neglected tropical diseases.

\section{Improve diagnosis}

Comparatively few studies have been carried out to improve diagnosis of PKDL. The conventional method for diagnosis of PKDL using light microscopy and skin specimens is not satisfactory and is especially frustrating for macular PKDL. ${ }^{8,9,24}$ Serological tests aimed at detection of antibodies against Leishmania in the blood do not help much since most PKDL patients have previously suffered from VL, and antibodies against Leishmania persist for years. However, it is useful for the diagnosis of PKDL patients who have not previously suffered from VL. Molecular tests such as detection of LD DNA by Leishmania-specific nested (Ln) polymerase chain reaction (PCR) in skin specimens are very sensitive for nodular and papular lesions and are satisfactory for the macular form of the disease. ${ }^{8,24}$ The problem with LnPCR is that there is a high probability of contamination and it is time consuming. The study by Verma et $\mathrm{al}^{33}$ showed that quantitative PCR using skin specimens and Taqman probe is very promising for the diagnosis of PKDL. However, its high cost limits its implementation by public health systems. Research for the development of antigen-detection tests from skin specimens or in blood/urine and fluorescence-based diagnostic tools using skin specimens are highly desired to develop cost-effective methods for diagnosis of PKDL.

\section{Improve treatment}

Safe and highly effective drugs are urgently needed for the treatment of PKDL cases especially in the Indian subcontinent. Among the new anti-leishmanial drugs (miltefosine, liposomal amphotericin B, paromomycin, and a combination of any two), only liposomal amphotericin $\mathrm{B}\left(\mathrm{AmBisome} \mathrm{e}^{\circledR}\right)$ and miltefosine monotherapy have been tested for their efficacy in the treatment of PKDL..$^{10,34,35}$ Therapy with liposomal amphotericin B resulted in different results in Sudan and Bangladesh. In Sudan, Musa et al ${ }^{10,35}$ reported promising results with AmBisome monotherapy at a dose of $2.5 \mathrm{mg} / \mathrm{kg} /$ day for 20 days. It showed $83 \%$ of cure rate in persistent PKDL patients who were resistant to SSG therapy. Another advantage of this treatment regimen is its comparatively shorter duration of treatment, which is 20 days, as well as its safety. However, the study enrolled only 12 PKDL patients, which is relatively small in size and necessitates further replication of this treatment regimen with a large number of patients to make a definite conclusion. Conversely, Médecins Sans Frontières, Holland program in Fulbaria, Bangladesh, found 34\% complete cure within 12 months of treatment with liposomal amphotericin B using a different dose schedule. ${ }^{10}$

Miltefosine (hexadecylcholine, $\mathrm{C}_{12} \mathrm{H}_{46} \mathrm{NO}_{4} \mathrm{P}$ ) is a phospholipid derivative, structurally related to a phospholipid component of the cell membrane. ${ }^{36}$ Its anti-leishmanial action was discovered by Croft et al in 1987. ${ }^{36}$ Following this discovery, miltefosine had been widely studied for its oral use as an anti-leishmanial drug at a dose of $2.0-2.5 \mathrm{mg} / \mathrm{kg} / \mathrm{day}$ for 4 weeks. It was highly effective against Indian VL with a cure rate of $83 \%-94 \%{ }^{37,38}$ Based on these studies in 2007 , miltefosine had been adapted for the treatment of VL for both adults and children ( $>2$ years old) by the national VL elimination program in Bangladesh, India, and Nepal.

In 2004, Soto et a ${ }^{39}$ reported its efficacy for the treatment of cutaneous leishmaniasis caused by Leishmania (Viannia) 
panamensis in Colombia. Miltefosine was also reported to be highly effective against cutaneous leishmaniasis by several other studies. ${ }^{40,41}$ However, in these studies, subjects were mostly adults and treatment with miltefosine was for 1 month.

Following the reports of treatment of cutaneous leishmaniasis with miltefosine, Belay et $\mathrm{al}^{42}$ and Rihl et al ${ }^{43}$ almost simultaneously reported one and two cases of PKDL HIV coinfection successfully treated with miltefosine in 2005. In the following year, Sundar et al reported the first case of PKDL unresponsive to SSG, but successfully treated with miltefosine. ${ }^{44}$ In subsequent years, several other studies have reported successful treatment of PKDL with miltefosine. ${ }^{45,46}$ Among these reports, only the study reported by Modak et al in 2010 included two children out of a total of six patients, who were treated successfully with miltefosine for 8 weeks. ${ }^{34}$ Ramesh et $\mathrm{al}^{47}$ found that adult PKDL patients could be treated with miltefosine daily for 8-12 weeks at a dose of $50 \mathrm{mg}$, three times a day with a cure rate of $96 \%$. Data from randomized control trials with miltefosine for PKDL were not available until January 2013 when Sundar et al showed that 12 weeks treatment with miltefosine for PKDL in adults was superior to 8 weeks treatment. ${ }^{48}$ The efficacy was $76 \%$ and $81 \%$ for 8 weeks and 12 weeks treatment regimens, respectively. So far, this is the only randomized clinical trial for the treatment of PKDL with miltefosine monotherapy for 12 weeks. However, the efficacy and safety of 12 weeks miltefosine treatment in children are unknown. A recent study in Bangladesh showed that children and adolescents are the most common victims of PKDL. ${ }^{3}$ Again further studies/trails are needed to confirm the efficacy and safety of miltefosine for PKDL in children. An open trial is under way in Bangladesh and its results will be available very soon (our study). We are also planning another study to investigate the safety and efficacy of 12 weeks miltefosine treatment in children and adolescents in Bangladesh.

Combination therapy is another attractive option for the treatment of PKDL. Ramesh et $\mathrm{al}^{49}$ reported two cases treated with amphotericin B and miltefosine. Combination therapy has the advantage of reducing the dose and duration to ensure better tolerance and compliance. The combination therapy of AmBisome plus miltefosine, AmBisome plus paromomycin, and miltefosine plus paromomycin has already been shown to be very safe and effective for VL. ${ }^{50}$ The efficacy and duration of combination therapy against PKDL are yet to be established.

Fexinidazole is a new oral drug. It is a 2 -substituted 5-nitroimidazole formulation. Currently, its efficacy and safety for VL is under trial in Sudan. ${ }^{51}$ If it is successful, this drug could also be tested for its efficacy against PKDL.

\section{Prevention of PKDL}

Prevention is better than cure. It follows that effort must be concentrated on the prevention of PKDL, which develops after treatment of VL. Unfortunately, except for SSG, the incidence of PKDL following any other treatment regimen either as monotherapy or combination therapy has not yet been systematically studied. Studies on the incidence of PKDL following different treatment regimens should be undertaken so as to identify better treatments associated with the least incidence of PKDL and without compromising its efficacy and safety against VL. Prospective active surveillance of cured VL patients is needed and patients treated under different drugs are ideal resources for eventual prospective surveillance. A recent study in India showed that treatment of VL with AmBisome at a total dose $20 \mathrm{mg} / \mathrm{kg}$ was associated with $0.3 \%$ incidence of PKDL in the cohort. ${ }^{14}$ This is far less than with SSG monotherapy, which was 19\% within 5 years after treatment. However, as PKDL patients seldom self-report to medical care facilities, active surveillance is needed to get the real estimate of PKDL incidence after treatment with a higher dose of AmBisome to make it comparable to the incidence of PKDL after other treatment regimens.

According to the available literature, one of the key points in the pathogenesis of PKDL is inadequate immune response to infection during and after the treatment of VL. Persistence of a mixed cellular immune response characterized by high levels of IL-10 and IFN $\gamma$ after complete treatment of VL is associated with development of PKDL. ${ }^{52}$ Therefore, immunoprophylaxis during the treatment of VL so as to enhance $\mathrm{Th}_{1}$ cellular immune responses along with a well-tuned immunoregulatory response to infection may prevent the development of PKDL after treatment of VL. This is supported by the findings of the study in Sudan in which PKDL treatment was substantially improved by adding alum/alum + BCG vaccine to the conventional treatment with SSG. ${ }^{31}$ LEISH F3 is the only US Food and Drug Administration (FDA)-approved vaccine in clinical trials for the prevention of VL. If it is found to be safe among the population where PKDL is common, then clinical trials with LEISH-F3 for the prevention of PKDL will be recommendable (personal communication with Dr Steve Reed, Director, Infectious Diseases Research Institute, Seattle, WA, USA).

In conclusion, there are several challenges for the management of PKDL. PKDL plays a crucial role as a reservoir 
during interepidemic transmission of VL especially in the Indian subcontinent where VL is only anthroponotic. However, it remains the most neglected issue among neglected diseases. In recent years, the burden of VL has substantially declined, which makes it appropriate to focus the efforts of donors, policymakers, and researchers on finding ways to effectively combat PKDL once and for all.

\section{Acknowledgment}

This work is not funded.

\section{Disclosure}

The authors report no conflicts of interest in this work.

\section{References}

1. Zijlstra EE, Musa AM, Khalil EAG, El Hassan IM, EI-Hassan AM. Post-kala-azar dermal leishmaniasis. Lancet Infect Dis. 2003;3:87-98.

2. Rahman KM, Islam S, Rahman MW, et al. Increasing incidence of Post-kala-azar dermal leishmaniasis in a population-based study in Bangladesh. Clin Infect Dis. 2010;50:73-76.

3. Islam S, Kenah E, Bhuiyan MA, et al. Clinical and immunological aspects of Post-kala-azar dermal leishmaniasis in Bangladesh. Am J Trop Med Hyg. 2013;89(2):345-353.

4. Bittencourt A, Silva N, Straatmann A, Nunes VL, Follador I, Badaró R. Post-kala-azar dermal leishmaniasis associated with AIDS. Braz J Infect Dis. 2003;7(3):229-233.

5. Zijlstra EE, Khalil EA, Kager PA, El-Hassan AM. Post-kala-azar dermal leishmaniasis in the Sudan: clinical presentation and differential diagnosis. Br J Dermatol. 2000;143(1):136-143.

6. Brahmachari UN. A new form of cutaneous leishmaniasis dermal leishmanoid. Indian Med Gaz. 1922;57:125.

7. Zijlstra EE, el-Hassan AM. Leishmaniasis in Sudan. Post-kala-azar dermal leishmaniasis. Trans R Soc Trop Med Hyg. 2001;95:S59-S76.

8. Mondal D, Nasrin KN, Huda MM, et al. Enhanced case detection and improved diagnosis of PKDL in a Kala-azar-endemic area of Bangladesh. PLoS Negl Trop Dis. 2010;4:ii:e832.

9. Addy M, Nandy A. Ten years of kala-zar in West Bengal, Part I. Did Post-kala-azar dermal leishmaniasis initiate the outbreak in 24-Parganas? Bull World Health Organ. 1992;70:341-346.

10. World Health Organization. Post-kala-azar dermal leishmaniasis: a manual for case management and control. Report of a consultative meeting; July 2-3, 2012; Kolkata, India. WHO/HTM/NTD/IDM/2013.1. Available from: http://apps.who.int/iris/bitstream/10665/78608/1/9789 241505215_eng.pdf. Accessed October 14, 2014.

11. Zijlstra EE, Alvar J. The Post Kala-azar Dermal Leishmaniasis Atlas: A manual for health workers. Geneva: World Health Organization; 2012. Available from: http://apps.who.int/iris/bitstream/10665/101164/1/978 9241504102_eng.pdf. Accessed October 3, 2014.

12. Croft SI. PKDL - a drug related phenomenon? Indian J Med Res. 2008;128:10-11.

13. Das VN, Pandey K, Verma N, et al. Short report: Development of Postkala-azar dermal leishmaniasis (PKDL) in miltefosine-treated visceral leishmaniasis. Am J Trop Med Hyg. 2009;8:336.

14. Burza S, Sinha PK, Mahajan R, et al. Post kala-azar dermal leishmaniasis following treatment with $20 \mathrm{mg} / \mathrm{kg}$ liposomal amphotericin B (Ambisome) for primary visceral leishmaniasis in Bihar, India. PLoS Negl Trop Dis. 2014;8(1):e2611.

15. World Health Organization. Regional Technical Advisory Group (RTAG) for the Kala-azar Elimination Programme. Report of the Fifth Meeting, Paro, Bhutan; September 17-19, 2013. SEA-CD-280.
16. Bern C, Chowdhury R. The epidemiology of visceral leishmaniasis in Bangladesh: prospects for improved control. Indian J Med Res. 2006;123(3):275-288.

17. Khan MH. Post-kala-azar dermal leishmaniasis (a review of 10 cases). Bangladesh Med Res Counc Bull. 1977;3:130-136.

18. Chowdhury AHK, Rahman J, Khan H, Tahir M. Post kala-azar dermal leishmaniasis (PKDL) study of 8 cases. Bangladesh Med J. 1988;17: 27-33.

19. Islam N, Rahman KM. Resurgence of visceral leishmaniasis in Bangladesh. Bull World Health Organ. 1983;61:113-116.

20. Masum MA, Alam B, Ahmed R. An epidemiological investigation of a kala-azar outbreak at Kalihati Upazila of Tangail District, Bangladesh. J Prev Soc Med. 1990;4-9:13-14.

21. Khan AM, Dutta P, Khan SA, et al. Kala-azar and Post-kala-azar dermal leishmaniasis. Assam, India. Emerg Infect Dis. 2014;20(3): 487-489.

22. Salotra P, Singh R. Challenges in the diagnosis of Post kala-azar dermal leishmaniasis. Indian J Med Res. 2006;123:295-310.

23. Srivastava P, Mehrotra S, Tiwary P, Chakravarty J, Sundar S. Diagnosis of Indian visceral leishmaniasis by nucleic Acid detection using PCR. PLoS One. 2011;6(4):e19304.

24. Adams ER, Versteeg I, Leeflang MM. Systematic review into diagnostics for Post-Kala-Azar Dermal Leishmaniasis (PKDL). J Trop Med. 2013;2013:150746.

25. World Health Organization. Report of a meeting of the WHO Expert Committee on the Control of Leishmaniasis; March 22-26, 2010, Geneva.

26. Singh S, Sivakumar R. Challenges and new discoveries in the treatment of Leishmaniasis. J Infect Chemother. 2004;10:307-315.

27. Thakur CP, Sinha GP, Pandey AK, et al. Do the diminishing efficacy and increasing toxicity of sodium stibogluconate in the treatment of visceral Leishmaniasis in Bihar, India, justify its continued use as a first-line drug? An observational study of 80 cases. Ann Trop Med Parasitol. 1998;92(5):561-569.

28. Maheshwari A, Seth A, Kaur S, et al. Cumulative cardiac toxicity of sodium stibogluconate and amphotericin b in treatment of kala-azar. Pediatr Infect Dis J. 2011;30:180-181.

29. Zanoni LZ, Brustoloni YM, Melnikov P, Consolo CEZ. Antimony containing drug and ECG abnormalities in children with visceral Leishmaniasis. Biol Trace Elem Res. 2009;132:35-40.

30. Thakur CP, Narain S, Kumar N, Hassan SM, Jha DK, Kumar A. Amphotericin B is superior to sodium antimony gluconate in the treatment of Indian post-kala-azar dermal leishmaniasis. Ann Trop Med Parasitol. 1997;91:611-616.

31. Musa AM, Khalil EA, Mahgoub FA, et al. Leishmaniasis Research Group/Sudan. Immunochemotherapy of persistent Post-kala-azar dermal leishmaniasis: a novel approach to treatment. Trans $R$ Soc Trop Med Hyg. 2008;102:58-63.

32. Huda MM, Hirve S, Siddiqui NA, et al. Active case detection in national visceral leishmaniasis elimination programs in Bangladesh, India, and Nepal: feasibility, performance and costs. BMC Public Health. 2012;12:1001.

33. Verma S, Bhandari V, Avishek K, Ramesh V, Salotra P. Reliable diagnosis of Post-kala-azar dermal leishmaniasis (PKDL) using slit aspirate specimen to avoid invasive sampling procedures. Trop Med Int Health. 2013;18(3):268-275.

34. Modak D, Basu A, Bhattacharya R, Pramanik N, Baribhula JN, Guha SK. Miltefosine in post-kala-azar dermal Leishmaniasis (PKDL). J Indian Acad Clin Med. 2010;11:199-203.

35. Musa AM, Khalil EA, Mahgoub FA, Hamad S, Elkadaru AM, El Hassan AM. Efficacy of liposomal amphotericin B (AmBisome) in the treatment of persistent post-kala-azar dermal leishmaniasis (PKDL). Ann Trop Med Parasitol. 2005;99:563-569.

36. Croft SL, Neal RA, Pendergast W, Chan JH. The activity of alkyl phosphorylcholines and related derivatives against Leishmania donovani. Biochem Pharmacol. 1987;36:2633-2636. 
37. Rahman M, Ahmed BN, Faiz MA, et al. Phase IV trial of miltefosine in adults and children for treatment of visceral leishmaniasis (kala-azar) in Bangladesh. Am J Trop Med Hyg. 2011;85(1):66-69.

38. Bhattacharya SK, Sinha PK, Sundar S, et al. Phase 4 trial of miltefosine for the treatment of Indian visceral leishmaniasis. J Infect Dis. 2007;196: 591-598.

39. Soto J, Ioledo J, Gutierrez P, et al. Treatment of American cutaneous leishmaniasis with miltefosine, an oral agent. Clin Infect Dis. 2001;33: E57-E61.

40. Machado PR, Ampuero J, Guimarães LH, et al. Miltefosine in the treatment of cutaneous leishmaniasis caused by Leishmania braziliensis in Brazil: a randomized and controlled trial. PLoS Negl Trop Dis. 2010;4(12):e912.

41. Chrusciak-Talhari A, Dietze R, Chrusciak Talhari C, et al. Randomized controlled clinical trial to access efficacy and safety of miltefosine in the treatment of cutaneous leishmaniasis caused by Leishmania (Viannia) guyanensis in Manaus, Brazil. Am J Trop Med Hyg. 2011;84: 255-260.

42. Belay AD, Asafa Y, Mesure J, Davidson RN. Successful miltefosine treatment of post-kala-azar-dermal Leishmaniasis occurring during antiretroviral therapy. Ann Trop Med Parasitol. 2006;100:223-227.

43. Rihl M, Stoll M, Ulbricht K, Bange F, Schmidt R. Successful treatment of post-kala-azar dermal Leishmaniasis in a HIV infected patient with multiple relapsing Leishmaniasis from Western Europe. J Infect. 2006;53:e25-e27.

44. Sundar S, Kumar K, Chakravarty J, et al. Cure of antimony-unresponsive Indian post-kala-azar dermal leishmaniasis with oral miltefosine. Trans R Soc Trop Med Hyg. 2006;100:698-700.
45. Ramesh V, Ansari NA, Jain RK, Salotra P. Oral miltefosine in the treatment of post-kala-azar dermal Leishmaniasis. Clin Exp Dermatol. 2007;33:103-104.

46. Khandpur S, Chaturvedi P, Kumar U, Khaitan BK, Samantaray JC, Sharma VK. Oral miltefosine in post-kala-azar dermal Leishmaniasis experience in three cases. Int J Dermatol. 2010;46:565-569.

47. Ramesh V, Katara GK, Verma S, Salotra P. Miltefosine as an effective choice in the treatment of post-kala-azar dermal Leishmaniasis. $\mathrm{Br} J$ Dermatol. 2011;165:411-414.

48. Sundar S, Sinha P, Jha TK, et al. Oral miltefosine for Indian post-kalaazar dermal Leishmaniasis: a randomized trial. Trop Med Int Health. 2013;18:96-100

49. Ramesh V, Avishek K, Sharma V, Salotra P. Combination therapy with amphotericin-B and miltefosine for post-kala-azar dermal leishmaniasis: a preliminary report. Acta Derm Venereol. 2014;94:242-243.

50. Sundar S, Sinha PK, Rai M, et al. Comparison of short-course multidrug treatment with standard therapy for visceral leishmaniasis in India: an open-label, non-inferiority, randomised controlled trial. Lancet. 2011;377(9764):477-486

51. Phase II proof of concept trial to determine efficacy of fexinidazole in visceral leishmaniasis patients in Sudan; 2014. Available from: http:// clinicaltrials.gov/show/NCT01980199.

52. Mondal D, Khan MG. Recent advances in post-kala-azar dermal leishmaniasis. Curr Opin Infect Dis. 2011;24(5):418-422.
Research and Reports in Tropical Medicine

\section{Publish your work in this journal}

Research and Reports in Tropical Medicine is an international, peerreviewed, open access journal publishing original research, case reports, editorials, reviews and commentaries on all areas of tropical medicine, including: Diseases and medicine in tropical regions; Entomology; Epidemiology; Health economics issues; Infectious disease; Laboratory

\section{Dovepress}

science and new technology in tropical medicine; Parasitology; Public health medicine/health care policy in tropical regions; and Microbiology. The manuscript management system is completely online and includes a very quick and fair peer-review system. Visit http://www.dovepress. com/testimonials.php to read real quotes from published authors. 